\title{
Programmed Polyene Cyclization Enabled by Chromophore Disruption
}

\author{
Megan M. Solans, Vitalii S. Basistyi, James A. Law, Noah M. Bartfield, and James H. Frederich*
}

Department of Chemistry and Biochemistry, Florida State University, Tallahassee, Florida 30326, United States

Supporting Information Placeholder

ABSTRACT: A versatile polyene cyclization strategy was developed that exploits conjugated $\beta$-ionyl derivatives. Photomediated disruption of the extended $\pi$-system within these chromophores unveils a contra-thermodynamic polyene that engages in a Heck-type cyclization to afford [4.4.1]-propellanes. The connectivity of overbred polycycles generated from this process is controlled by the position of the requisite $\mathrm{C}-\mathrm{Br}$ bond. Thus, compared to conventional biomimetic polyene cyclization, this approach allows for complete control of regi-
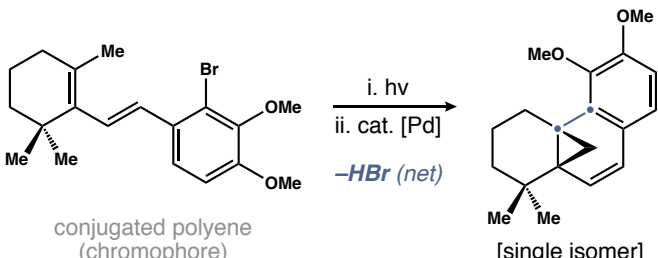
ochemistry and facilitates incorporation of both electron-rich and electron-deficient (hetero)aryl groups. This strategy was successfully applied to the total synthesis of taxodione and salviasperanol, two isomeric abietane-type diterpenes that previously could not be prepared along the same synthetic pathway.

Diterpenes are a rich source of biologically active molecular scaffolds. ${ }^{1,2}$ Members of this family harboring a fused [6-6-6] carbocyclic nucleus have gained significant attention for their valuable pharmacological profiles. ${ }^{3-5}$ This subgroup of natural products includes abietane, pimarane, and kaurane diterpenes, which all share copalyl diphosphate as a common biosynthetic progenitor (Figure 1). ${ }^{6}$ Although the skeletal diversity of these diterpenes is localized within the C-ring, each motif is further differentiated through late-stage oxidative tailoring. ${ }^{7}$ This added layer of complexity hinders the design of unified synthetic entry to this diverse collection of targets. Recent advances in synthetic biology have streamlined entry to this subgroup from more accessible terpenoid starting points. ${ }^{8}$ In contrast, polyene cyclization provides a generalized platform for de novo diterpene synthesis. This bioinspired strategy seeks to reorganize isolated polyenes (1) to polycycles (2) through a series of concerted $\mathrm{C}-\mathrm{C}$ bond formations. ${ }^{9}$ Both cationic and radical reaction modalities are known, as are enantioselective adaptations catalyzed by Lewis acids, ${ }^{10}$ organocatalysts, ${ }^{11}$ and transition metals. ${ }^{12}$ The outcomes of polyene cyclization are predictable and governed by explicit aspects of alkene stereochemistry and electronics. ${ }^{9 \mathrm{a}}$ This powerful feature is often exploited in total synthesis $;{ }^{9 \mathrm{c}}$ however, it also makes it difficult to extend this strategy to diversified substrates (e.g. 1a-1c) that deviate from biogenic isoprenoid precursors. ${ }^{13,14}$

Inspired by the photobiology of vitamin A, we envisioned a different polyene cyclization strategy. In place of isolated polyene 1, we targeted conjugated $\beta$-ionyl derivative 3 . Following Buchi's investigations on the photoisomerization of $\beta$-ionone to $Z$-retro- $\gamma$-ionone, ${ }^{15}$ we reasoned that diene 4 could be unveiled from 3 via a photoinduced [1,5]-hydride shift. The bifurcated $\pi$-systems in $\mathbf{4}$ could then be connected via a Heck cyclization, where the position of the $\mathrm{C}-\mathrm{X}$ bond would guide site-specific incorporation of the aryl ring. We predicted that a 6-exo-trig pathway to generate alkyl metal species A would be favored, ${ }^{16}$ thereby providing an avenue to modify the oxidation-state of $\mathrm{C} 20$, most directly by cyclization to propellane 5 . Fragmentation of this overbred species was viewed as handle for skeletal editing. Moreover, this sequence would allow 5 to be assembled from interchangeable fragments. We expected these features to not only expedite entry to diterpene natural products, but also simplify the design of tailored compositions
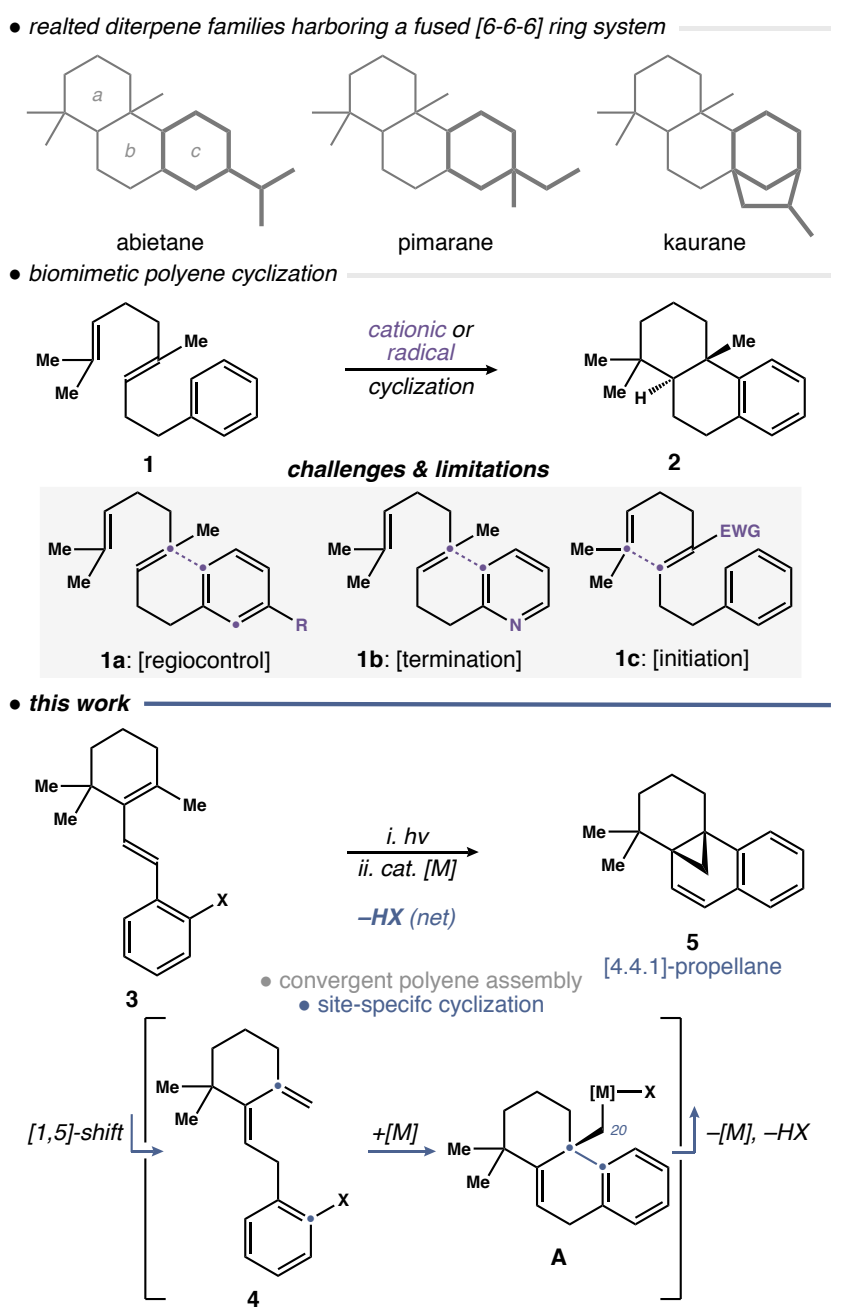

Figure 1. A polyene cyclization strategy enabled by photoinduced disruption of the extended $\pi$-system within 3 .

of matter that are beyond the reach of semi-synthesis. Herein, we report the successful implementation of this programmed polycyclization strategy and highlight its utility for the synthesis of canonical and rearranged abietane diterpenes. ${ }^{17}$ 
Table 1. Photoisomerizaiton of $\beta$-ionyl derivatives $3^{a, b}$

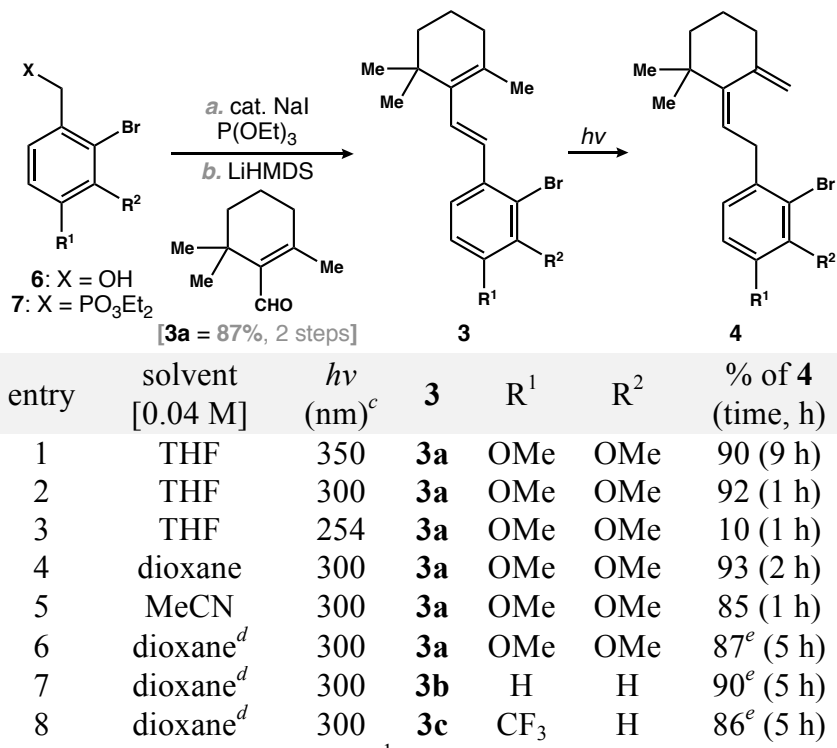

${ }^{a}$ Yields were determined by ${ }^{1} \mathrm{H}$ NMR using $\mathrm{Me}_{2} \mathrm{SO}_{2}$ as an internal standard. ${ }^{b}$ Solvent was degassed by successive freeze-pumpthaw cycles. ${ }^{c}$ Reactions were carried out in a Rayonet photoreactor maintained at $35^{\circ} \mathrm{C} .{ }^{d}[4]=0.2 \mathrm{M}^{e}{ }^{e}$ Isolated yield after purification by silica gel chromatography.

We began by examining the photoisomerization of $\beta$-ionyl derivatives 3. While the photosensitized geometric isomerization of $\beta$-ionyl species is established, ${ }^{18}$ positional alkene isomerization via a [1,5]-hydride shift is an underexplored pathway observed upon direct irradiation. ${ }^{19}$ Thus, it was not clear if this reaction would be broadly synthetically useful. To address this concern, a set of electronically differentiated photosubstrates 3 were prepared from benzylic alcohols 6 via conversion to aryl phosphonates 7 and olefination with $\beta$-cyclocitral. ${ }^{20}$ As shown in Table 1, we were pleased to find that irradiation of $\mathbf{3 a}\left(\mathrm{R}^{1}\right.$, $\mathrm{R}^{2}=\mathrm{OMe}$ ) with $350 \mathrm{~nm}$ light in deoxygenated THF provided $Z$-diene $4 a$ in $90 \%$ yield (entry 1$){ }^{21}$ The reaction time was reduced to $1 \mathrm{~h}$ using $300 \mathrm{~nm}$ light (entry 2). In contrast, 254 $\mathrm{nm}$ light resulted in decomposition (entry 3 ). Dioxane and MeCN gave similar results to THF (entries 4 and 5); however, the reaction rate slowed as the concentration of $\mathbf{3 a}$ increased. Guided by these observations, we developed a general procedure using $300 \mathrm{~nm}$ light in dioxane $(0.2 \mathrm{M})$ that provided $4 \mathbf{a}$ in $87 \%$ yield after $5 \mathrm{~h}$ (entry 6 ). This transformation was remarkably efficient, with no evidence of other products by ${ }^{1} \mathrm{H}$ NMR. Moreover, these mild conditions were extended to differentiated cyclization substrates $\mathbf{3 b}\left(\mathrm{R}^{1}, \mathrm{R}^{2}=\mathrm{H}\right)$ and $\mathbf{3} \mathbf{c}\left(\mathrm{R}^{1}=\mathrm{CF}_{3}, \mathrm{R}^{2}\right.$ $=\mathrm{H})$ to generate $\mathbf{4 b}(90 \%$ yield $)$ and $\mathbf{4 c}(86 \%$ yield $)$ as single isomers, respectively (entries 7 and 8).

The isomerization of $\mathbf{3 a}$ was explored in detail (Scheme 1). Analysis of a test reaction ( $h v=300 \mathrm{~nm}, 0.1 \mathrm{M}$ in THF) by ${ }^{1} \mathrm{H}$ NMR revealed initial isomerization of the exocyclic alkene to generate a mixture of $(E)-\mathbf{3 a}$ and $(Z)-\mathbf{3 a}$. These species were present in a 1:1 ratio after $1 \mathrm{~h}$, alongside 4a. After $2 \mathrm{~h},(E)-\mathbf{3 a}$ was consumed and a mixture of $(Z)-\mathbf{3 a}$ and $\mathbf{4 a}$ persisted until the reaction was complete. We prepared $(Z)-\mathbf{3 a}$ independently and found that the photochemistry of this isomer was indistinguishable from $(E)-3 \mathbf{a}^{22}$ Thus, the initial geometry of the exocyclic alkene was inconsequential. In contrast, $4 \mathbf{a}$ did not react with $300 \mathrm{~nm}$ light, yet reverted to $(E)-\mathbf{3 a}$ in $>95 \%$ yield when heated to $150{ }^{\circ} \mathrm{C}$ via a thermal (retro) [1,5]-hydride shift. These results indicate that $\mathbf{4 a}$ is metastable intermediate that likely
Scheme 1. Chromophore disruption in the photoisomerization of $3 \mathbf{a}$ to $4 \mathbf{a}^{a}$

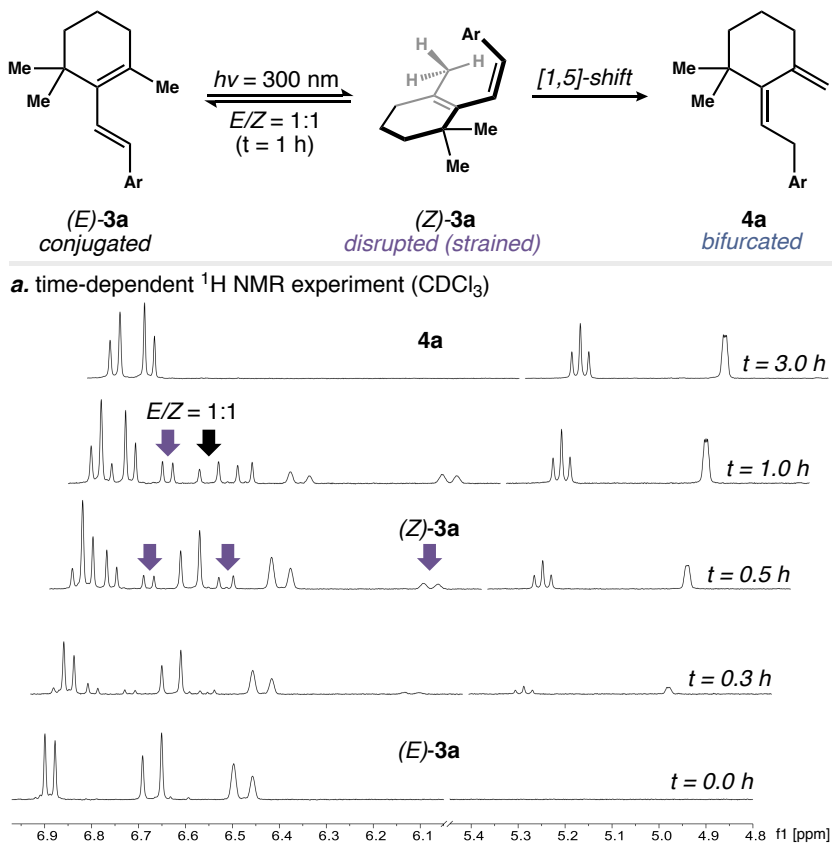

b. UV-vis spectra of independently prepared (E)-3a, (Z)-3a, and $\mathbf{4 a}$ in THF

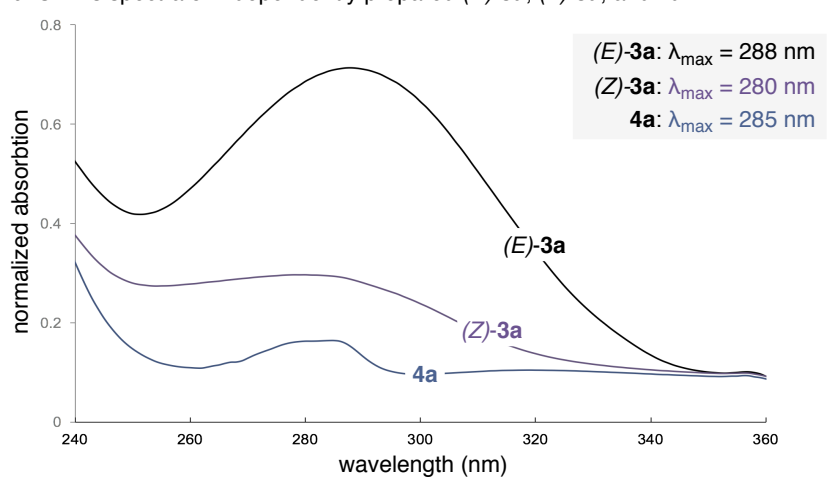

${ }^{a}$ Structure 4a reverted directly to $(E)-3 \mathbf{a}$ at $150{ }^{\circ} \mathrm{C}$.

accumulates because disruption of the extended $\pi$-system prevents re-excitation. This conclusion was supported by analyzing the absorption spectra of $(E)-\mathbf{3 a},(Z)-\mathbf{3 a}$, and $\mathbf{4 a}$ in THF. These data revealed significant hypochromic shifts for $(Z)-\mathbf{3 a}$ and $\mathbf{4 a}$ relative to $(E)-\mathbf{3 a}$, which is consistent with impaired conjugation. Notably, the $\pi$-system in (Z)-3a is distorted by allylic strain, which also restricts interconversion to the s-trans conformer. ${ }^{23}$ This same steric effect may also facilitate a photochemical [1,5]-hydride shift within $(Z)-\mathbf{3 a}$ to give $\mathbf{4 a}$, a process that requires an otherwise difficult-to-achieve antarafacial transition state. ${ }^{24}$

Our next goal was to execute the proposed Heck reaction within 4a. After some experimentation, we identified the combination of $\mathrm{Pd}_{2} \mathrm{dba}_{3} / \mathrm{P}(t-\mathrm{Bu})_{3}(1: 1 \mathrm{Pd} / \mathrm{P})$ and $\mathrm{Cy}_{2} \mathrm{NMe}$ described by $\mathrm{Fu}$ to be the most effective. ${ }^{25}$ Exposure of $\mathbf{4 a}$ to this catalyst system in dioxane at $85{ }^{\circ} \mathrm{C}$ gave $\mathbf{5 a}$ in $93 \%$ yield. As shown in Scheme 2, the photoisomerization and Heck cyclization were merged into a single operation. This more convenient process afforded 5a in 76\% yield from 3a on gram-scale. ${ }^{26}$ Moreover, this process was effective across a range of cyclization precursors (3b-3k). Accordingly, C-ring variants 3b-3e with peripheral substituents at every position around the aryl nucleus were tolerated. By design, a single regioisomer of $\mathbf{5}$ was obtained in 
Scheme 2. Cyclization of $\beta$-ionyl substrates 3: Scope and limitations. $^{a}$

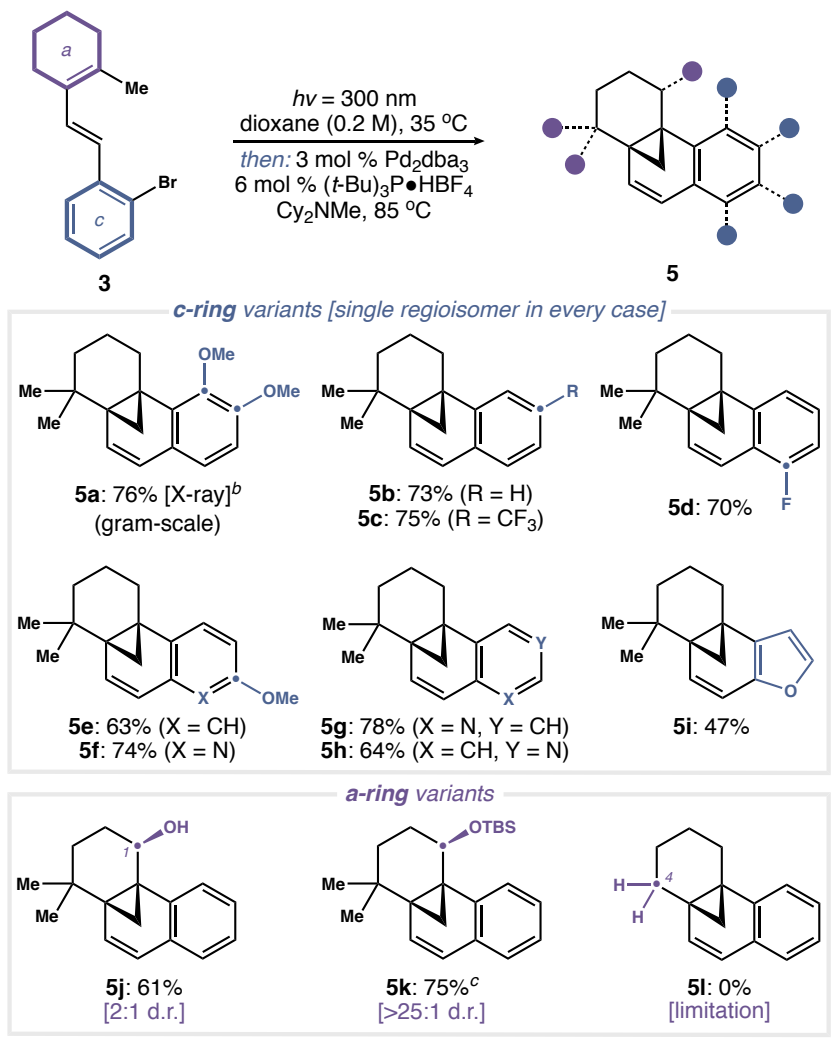

${ }^{a}$ Unless otherwise noted, reactions were carried out on $0.3 \mathrm{mmol}$ scale. Reported yields are based on isolated product after purification. ${ }^{b}$ A FEP-tubing spindle was used for the photochemistry (see the Supporting Information for details). ${ }^{c}$ Relative stereochemistry was established by single crystal X-ray diffraction.

every case. In addition, the reaction was effective for electronrich (5a and 5e), non-activated (5b), and electron-deficient (5c and $\mathbf{5 d}$ ) substrates. This feature allowed us to prepare variants of $\mathbf{5}$ harboring $\pi$-deficient (5f-5h) and $\pi$-excessive (5i) heterocyclic rings. Modifications of the A-ring were also investigated. For example, the addition of an alcohol at $\mathrm{C} 1$ provided $\mathbf{5} \mathbf{j}$ in $2: 1$ d.r. (61\% yield). ${ }^{27}$ This selectivity was readily improved to $>25: 1$ d.r. in $\mathbf{5 k}$ ( $75 \%$ yield) when a bulky silyl protecting group was included. In contrast, propellane 5l lacking a gemdimethyl group at $\mathrm{C} 4$ was a limitation. In this case, photolysis of $\mathbf{3 l}$ did not produce isomer $4 \mathbf{l}$. Instead, $E \rightarrow Z$ isomerization of the exocyclic alkene was the exclusive reaction. This outcome demonstrates the importance of allylic strain introduced by the gem-dimethyl group in the [1,5]-hydride shift process.

Having established the proposed photoinduced polycyclization cascade, we turned our attention to examining its utility in target-oriented synthesis. As shown in Scheme 3, we selected abietane diterpenes for this purpose. Our interest in this diterpene family was inspired by taxodione (8), a novel inhibitor of farnesyl diphosphate synthase. ${ }^{28}$ Among the reported syntheses of $\mathbf{8},{ }^{29}$ an 11-step route featuring biomimetic polycyclization is the most concise. ${ }^{30}$ This work provided a valuable benchmark. We also targeted salviasperanol (9), an isomer of 8 distinguished by a [6-7-6] icetexane skeleton. ${ }^{31}$ In Nature, this rearranged abeo-abietane framework is prepared from an abietane via an oxidation-enabled Wagner-Meerwein rearrangement. ${ }^{32}$ Nevertheless, 9 is not readily accessible using polyene cyclization. As a result, existing total syntheses of $\mathbf{8}$ and $\mathbf{9}$ treat each
Scheme 3. Synthetic entry to common intermediate 11.
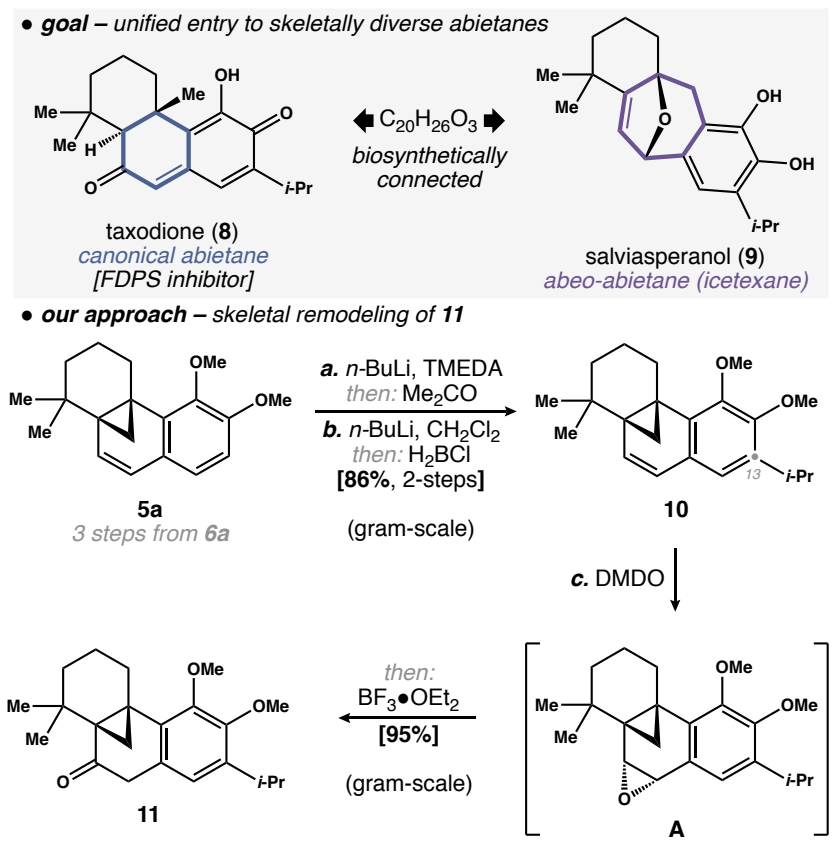

carbocyclic framework as an independent problem. ${ }^{33}$

To evaluate the utility of our strategy, we set out to develop a unified entry point to $\mathbf{8}$ and $\mathbf{9}$ via late-stage diversification of overbred abietane 11. This common intermediate was prepared on gram-scale from 5a via ortho-lithiation and trapping with acetone. Deoxygenation of the resultant alcohol using Kabalka's protocol provided $\mathbf{1 0}$ in $86 \%$ overall yield. ${ }^{34}$ The olefin in 10 was then reacted with DMDO to give epoxide A, a transient species that smoothly rearranged to ketone $\mathbf{1 1}$ in $95 \%$ yield upon exposure to $\mathrm{BF}_{3} \bullet \mathrm{OEt}_{2}$.

A total synthesis of $( \pm$ )-taxodione $(\mathbf{8})$ was achieved from 11 via ring-opening of the activated cyclopropyl ketone (Scheme 4). In this case, 11 was reacted with excess $\mathrm{TMSCl}$ and $\mathrm{NaI}$ in MeCN. ${ }^{35}$ Following an aqueous workup, this protocol provided iodide 12 in $>95 \%$ yield. It was best not to purify this sensitive intermediate. Instead, alkyl iodide 12 was directly reduced with zinc powder to furnish 13 in $82 \%$ yield. Deprotection of the aryl ethers with $\mathrm{BBr}_{3}$ then unveiled the corresponding catechol, which rapidly to oxidized $\mathbf{8}$ in $93 \%$ yield upon exposure to $\mathrm{SiO}_{2}$ and oxygen. ${ }^{36}$ As such, this approach provided $( \pm)-\mathbf{8}$ in 9 total steps and $41 \%$ overall yield from commercially available fragments $\mathbf{6 a}$ and $\beta$-cyclocitral.

Intermediate 11 was also diverted to $( \pm)$-salviasperanol (9). In this context, alkyl iodide $\mathbf{1 2}$ was generated in situ as before and then treated with $\mathrm{Ag}(\mathrm{TFA})$ to induce a biomimetic skeletal rearrangement. ${ }^{32}$ Under dilute conditions $(0.05 \mathrm{M})$, this reaction provided enone $\mathbf{1 4}$ in $63 \%$ yield alongside an 8:1 mixture of isomers 15a and 15b. Mixtures of 15 were recycled to 14 in two steps. In this case, Mukaiyama hydration of $\mathbf{1 5}$ gave alcohol 16 as a single diastereomer in $83 \%$ yield. Subsequent dehydration of 16 using Burgess reagent (17) afforded 14 in 70\% yield. Alternatively, a formal synthesis of $( \pm)-9$ was completed by reducing 14 with $\mathrm{LiAlH}_{4}$. Dehydration of the resultant allylic alcohol with 17 furnished diene $\mathbf{1 8}$ in 59\% yield over two steps. Notably, Sarpong and co-workers previously elaborated 18 to $9 .{ }^{33 \mathrm{a}}$ Therefore, 9 was formally prepared in 9 total steps ( $21 \%$ overall yield) and, for the first time, along the same synthetic pathway as abietane $\mathbf{8}$. 
Scheme 4. Diverted total synthesis of $( \pm)$-taxodione (8) and formal synthesis of $( \pm)$-salviasperanol (9) from ketone 11. - ( \pm )-taxodione (8)

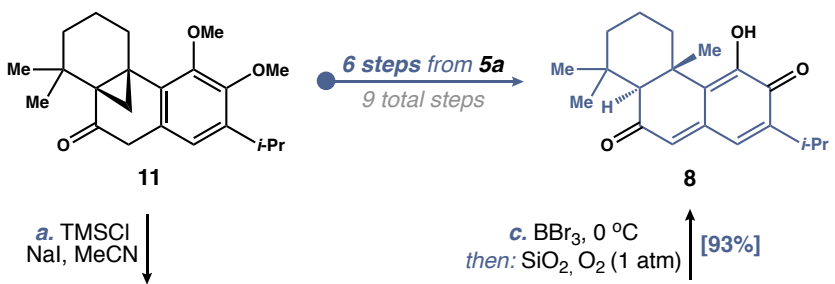

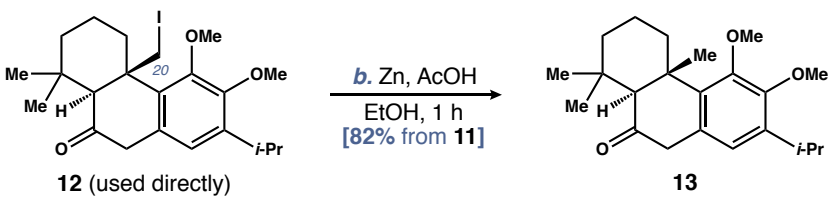

- ( \pm )-salviasperanol (9) — icetexane diterpene
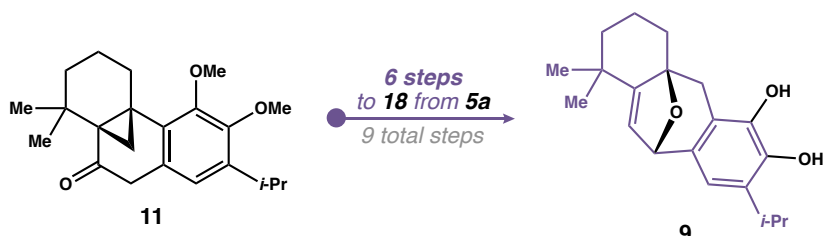

$$
\begin{array}{c|c}
\text { a. TMSCl } \\
\mathrm{Nal}, \mathrm{MeCN}
\end{array} \mid \begin{gathered}
\text { then: } \mathrm{Ag}(\mathrm{TFA}) \\
{[63 \%]}
\end{gathered}
$$
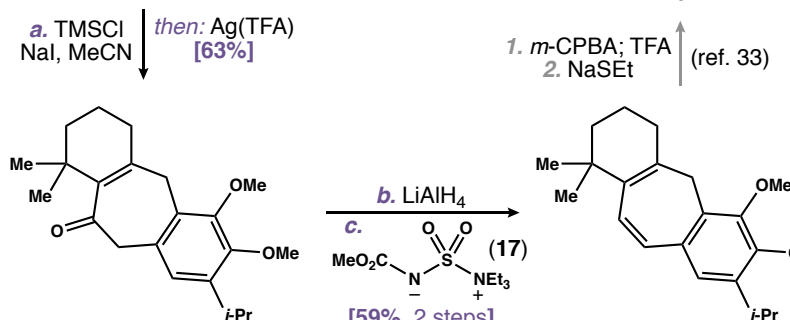

14

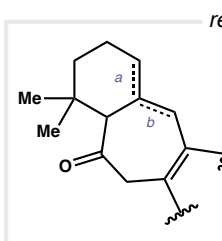

15: [30\% from 11$]$ $(a: b=8: 1)$ recycling undesired isomers 15 to 14

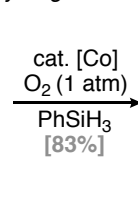

16: single isomer (X-ray)

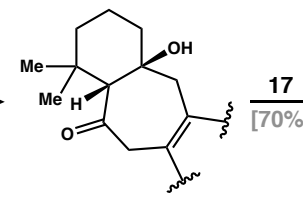

Skeletal remodeling of $\mathbf{1 1}$ also supported entry to unprecedented abeo-abietane scaffolds (Scheme 5). For example, exposure of 11 to $\mathrm{BF}_{3} \bullet \mathrm{OEt}_{2}$ in toluene at $80{ }^{\circ} \mathrm{C}$ afforded isomeric [6-7-6] ring system 19 in $95 \%$ yield. We suspect that that 19 is derived of cation $\mathbf{A}$, which itself arises from a (net) [1,3]alkyl shift within the initial Lewis adduct of 11. ${ }^{37}$ Alternatively, irradiation of $11(h v=254 \mathrm{~nm})$ in the presence of $\mathrm{Et}_{3} \mathrm{~N}$ generated [4.4.1] bicyclic motif 20, putatively via ketyl radical B. ${ }^{38}$ This carbocycle is reminiscent of the cyclocitrinols, ${ }^{39}$ and was available at a higher oxidation state from 10. In this case, $\mathbf{1 0}$ was reacted with NIS in aqueous THF to give halohydrin C. Upon exposure to $\operatorname{Ag}(\mathrm{TFA})$, this reactive species furnished alcohols 21 and 22 in $82 \%$ yield (1:1 ratio). We found that $\mathbf{2 1}$ isomerized to $\mathbf{2 2}$ in the presence of $\mathrm{TsOH}$, putatively via cation D. Alternatively, 22 was isolated in $82 \%$ yield when $\mathrm{MeCN}$ was used in place of THF. Collectively, these non-canonical abietanes illustrate the structural diversity that can be achieved by controlled fragmentation of the propellane within $\mathbf{5 a}$.

In summary, we established an atypical polyene cyclization strategy that converts easily accessible $\beta$-ionyl derivatives into polycyclic [4.4.1]-propellanes. Unlike canonical biomimetic
Scheme 5. Entry to diversified non-natural abeo-abietanes via skeletal remodeling of propellanes $\mathbf{1 0}$ and $\mathbf{1 1}$.

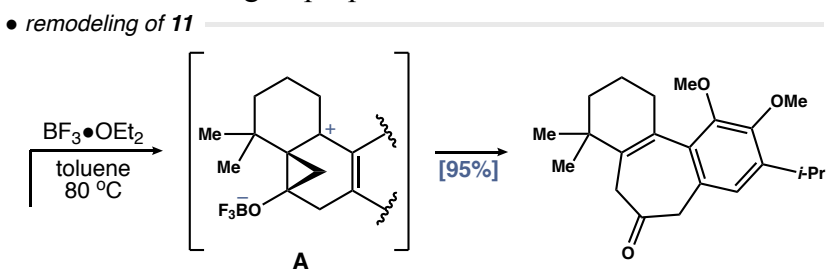

11

19 (X-ray)
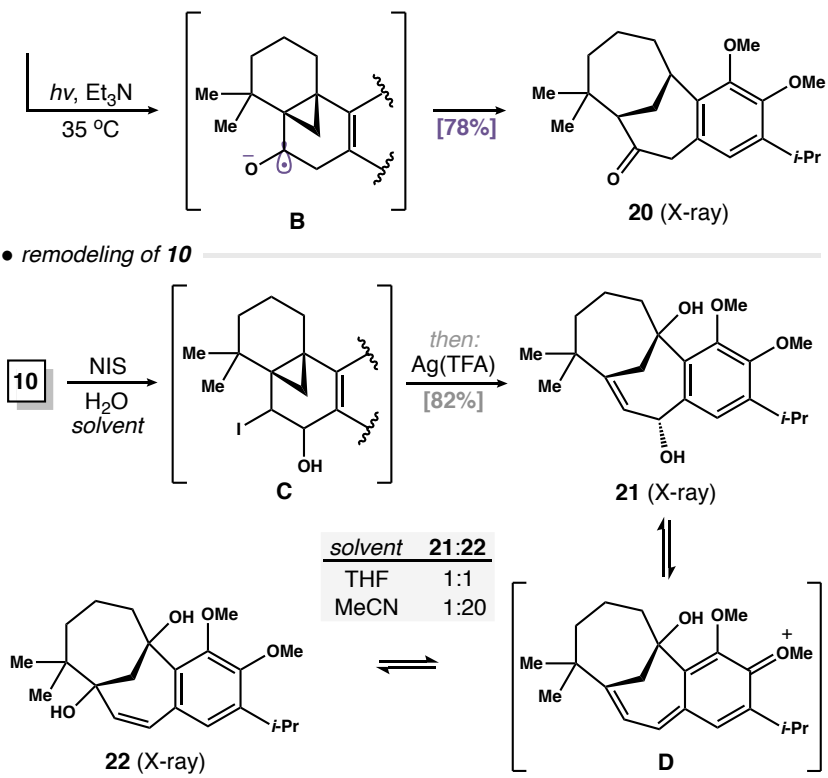

polyene cyclization, this strategy allows for complete control of regiochemistry and tolerates a range of electron-rich and electron-deficient (hetero)aryl groups. The strained polycycles produced from this chemistry were elaborated to diterpene architectures via late-stage skeletal remodeling. This powerful feature was highlighted in a unified synthesis of taxodione (8) and salviasperanol (9) that is concise, modular, and amenable to asymmetric induction in future iterations. Alternative diversification pathways were explored, and these generated a collection of non-canonical abeo-abietane scaffolds that would be difficult or impossible to prepare using conventional biomimetic logic. As a result, this terpene assembly strategy appears to be well-suited for applications in both target- and diversityoriented synthesis.

\section{ASSOCIATED CONTENT}

\section{Supporting Information}

The Supporting Information is available free of charge on the ACS Publications website.

Experimental procedures, supplemental figures, and characterization data for all new compounds (PDF)

\section{Accession Codes}

Supplementary crystallographic data for this paper can be found under the CCDC accession codes: 2129320 (5a), 21229321 (16), 2129323 (19), 2129319 (20), 2129322 (21), and 2129324 (22). These data can be obtained free of charge from the Cambridge Crystallographic Data Centre (CCDC) at www.ccdc.cam.ac.uk.

\section{AUTHOR INFORMATION}




\section{Corresponding Author}

James H. Frederich - Florida State University, Department of Chemistry and Biochemistry, Tallahassee, FL 32306, United States.

E-Mail: frederich@chem.fsu.edu

\section{Authors}

Megan M. Solans - Florida State University, Department of Chemistry and Biochemistry, Tallahassee, FL 32306, United States.

Vitalii S. Basistyi - Florida State University, Department of Chemistry and Biochemistry, Tallahassee, FL 32306, United States.

James A. Law - Florida State University, Department of Chemistry and Biochemistry, Tallahassee, FL 32306, United States.

Noah M. Bartfiled - Yale University, Department of Chemistry, New Haven, CT 06520, United States

\section{Notes}

The authors declare no competing financial interest

\section{ACKNOWLEDGMENT}

This work was funded by a Florida State University (FSU) Planning Grant (CRC-3425) and, in part, by the NIGMS branch of the NIH under award R01GM125926. We acknowledge Dr. Xinsong Lin (FSU) for his assistance with X-ray crystallography and mass spectrometry. We also thank Professor Joel Smith (FSU) for valuable discussions.

\section{REFERENCES}

(1) Cox-Georgian, D.; Ramadoss, N.; Dona, C.; Basu, C. Therapeutic and medicinal uses of terpenes. Medicinal Plants 2019, 333-359.

(2) Newman, D. J.; Cragg, G. M. Natural products as sources of new drugs over the nearly four decades from $01 / 1981$ to $09 / 2019$. J. Nat. Prod. 2020, 83, 770-803.

(3) Gonzales, M. A. Aromatic abietane diterpenoids: Their biological activity and synthesis. Nat. Prod. Rep. 2015, 32, 684-704.

(4) Reveglia, P; Cimmino, A.; Masi, M.; Nocera, P.; Berova, N.; Ellestad, G.; Evidente, A. Pimarane diterpenes: Natural source, stereochemical configuration, and biological activity. Chirality 2018, 30, $1115-1134$.

(5) Zhao, X.; Cacherat, B.; Hu, Q.; Ma, D. Recent advances in the synthesis of ent-Kaurane diterpenoids. Nat. Prod. Rep. 2022, 39, 119 138.

(6) (a) Tantillo, D. J. Biosynthesis via carbocations: Theoretical studies on terpene formation. Nat. Prod. Rep. 2011, 28, 1035-1053; (b) Hong, Y. J.; Tantillo, D. J. Formation of Beyerene, Kaurene, Trachylobane, and Atiserene diterpenes by rearrangements that avoid secondary carbocations. J. Am. Chem. Soc. 2010, 132, 5375-5386.

(7) Dibrell, S. E.; Tao, Y.; Reisman, S. E. Synthesis of complex diterpenes: Strategies guided by oxidation pattern analysis. Acc. Chem. Res. 2021, 54, 1360-1373.

(8) Zhang, X.; King-Smith, E.; Dong, L.-B.; Yang, L.-C.; Rudolf, J. D.; Shen, B.; Renata, H. Divergent synthesis of complex diterpenes via a hybrid oxidative approach. Science 2020, 369, 799-806.

(9) For reviews, see: Yoder, R. A.; Johnston, J. N. A case study on biomimetic total synthesis: Polyolefin carbocyclizations to terpenes and steroids. Chem. Rev. 2005, 105, 4730-4756; (b) Ungarean, C. N.; Southgate, E. H.; Sarlah, D. Enatioselective polyene cyclizations. Org. Biomol. Chem. 2016, 14, 5454-5467; (c) Barrett, A. G. M., Ma, T.; Mies, T. Recent developments in polyene cyclization and their applications in natural product synthesis. Synthesis 2019, 51, 67-82; (d) Feilner, J. M.; Haut, F.-L.; Magauer, T. Beyond the isoprene pattern: Bifunctional polyene cyclizations. Chem. Eur. J. 2021, 27, 7017-7021.

(10) For select examples, see: (a) (c) Ishihara, K.; Nakamura, S.; Yamamoto, H. The first enantioselective biomimetic cyclization of polyprenoids. J. Am. Chem. Soc. 1999, 121, 4906-4907; (b) Zhao, Y.-
J.; Chng, S.-S.; Loh, T.-P. Lewis Acid-promoted intermolecular acetal-initiated cationic polyene cyclizations. J. Am. Chem. Soc. 2007, $129,492-493$.

(11) For select examples, see: (a) Rendler, S.; MacMillan, D. W. C. Enantioselective polyene cyclization via organo-SOMO catalysis. $J$. Am. Chem. Soc. 2010, 132, 5027-5029; (b) Plamondon, S. J.; Warnica, J. M.; Kaldre, D.; Gleason, J. L. Hydrazide-catalyzed polyene cyclization: Asymmetric organocatalytic synthesis of cis-decalins. Angew. Chem., Int. Ed. 2020, 59, 253-258; (c) b) Kutateladze, D. A.; Strassfeld, D. A., Jacobsen, E. N. Enantioselective tail-to-head cyclizaitons catalyzed by dual-hydrogen-bond donors. J. Am. Chem. Soc. 2020, 142, 6951-6956.

(12) For select examples, see: (a) Sethofer, S. G.; Mayer, T.; Toste, F. D. Gold(I)-catalyzed enantioselective polycyclization reactions. $J$. Am. Chem. Soc. 2010, 132, 8276-8277; (b) Schaforth, M. A.; Sarlah, D.; Krautwald, S.; Carreira, E. M. Iridium-catalyzed enantioselective polyene cyclization. J. Am. Chem. Soc. 2012, 134, 20276-20278; (c) Cochrane, N. A.; Nguyen, H.; Gagne, M. R. Catalytic enantioselective cyclization and $\mathrm{C} 3$-fluorination of polyenes. J. Am. Chem. Soc. 2013, $135,628-631$.

(13) For representative examples of challenges encountered with 1a, see: (a) Grayfer, T. D.; Retailleau, R.; Dood, R. H.; Dubois, J.; Cariou, K. Chemodivergent, tunable, and selective iodine(III)-mediated bromo-functionalizations of polyprenoids. Org. Lett. 2017, 19, 47664769; (b) Ishihara, K.; Ishibashi, H.; Yamamoto, H. Enantioselective biomimetic cyclization of homo(polyprenyl)arenes. A new entry to $(+)$-podpcarpa-8,11,13-triene diterpenoids and (-)-tetracyclic polyprenoid of sedimentary origin. J. Am. Chem. Soc. 2001, 123, 15051506.

(14) Electronically deactivated polyenes (e.g. 1b and 1c) are challenging substrates for canonical polyene cyclization: (a) Vrubliauskas, D.; Vanderwal, C. D. Cobalt-catalyzed hydrogen-atom transfer induces bicyclizations that tolerate electron-rich and electron deficient intermediate alkenes. Angew. Chem., Int. Ed. 2020, 59, 6115-6121. (b) Snyder, S. A.; Treitler, D. S. $\mathrm{Et}_{2} \mathrm{SBr} \bullet \mathrm{SbCl}_{5} \mathrm{Br}$ : An effective reagent for direct bromonium-induced polyene cyclizations. Angew. Chem., Int. Ed. 2009, 48, 7899-7903.

(15) Büchi, G.; Yang, N. C. Light-catalyzed organic reactions. VI. The isomerization of some dienones. J. Am. Chem. Soc. 1957, 79, $2318-2323$

(16) Limura, S.; Overman, L. E.; Paulini, R.; Zakarian, A. Enantioselective total synthesis of guanacastepene $\mathrm{N}$ using an uncommon 7endo Heck cyclization as a pivotal step. J. Am. Chem. Soc. 2006, 128, 13095-13101.

(17) A creative polycyclization approach to abietane diterpenes was disclosed during the course of this study: (a) Vrubliauskas, D.; Gross, B, M.; Vanderwal, C. D. Stereocontrolled radical bicyclizations of oxygenated precursors enable short syntheses of oxidized abietane diterpenoids. J. Am. Chem. Soc. 2021, 143, 2944-2952; (b) Johnson, L. K.; Niman, S. W.; Vrubliauskas, D.; Vanderwal, C. D. Stereocontrolled synthesis and structural revision of plebeianol A. Org. Lett. 2021, 23, 9569-9573.

(18) (a) Naskar, S.; Chowdhury, S. R.; Mondal, S.; Maiti, D. K.; Mishra, S.; Das, I. Visible-light-activated divergent reactivity of dienones: Dimerization in neat conditions and regioselective $E$ to $Z$ isomerization in solvent. Org. Lett. 2019, 21, 1578-1582; (b) Livingstone, K.; Tenverge, M.; Pape, F.; Daniliuc, C. G.; Jamieson, C.; Gilmour, R. Photocatalytic $E \rightarrow Z$ isomerization of $\beta$-ionyl derivatives. Org. let. 2019, 21, 9677-9680.

(19) (a) de Mayo, P; Stothers, J. B; Yip, R. W. The irradiation of $\beta$ ionone. Can. J. Chem. 1961, 39, 2135-2137; (b) Ramamurthy, V.; Liu, R. S. H. Sigmatropic hydrogen migration and electrocyclization processes in compounds in the vitamin A series. Photochemistry of polyenes. J. Org. Chem. 1976, 41, 1862-1867.

(20) Ma, X.; Xu, Q.; Li, H.; Su, C.; Yu, L.; Zhang, X.; Cao, H.; Han, L.-B. Alcohol-based Michaelis-Arbuzov reaction: An efficient and environmentally-benign method for $\mathrm{C}-\mathrm{P}(\mathrm{O})$ bond formation. Green Chem. 2018, 20, 3408-3413.

(21) The endoperoxide arising from photooxidation of $\mathbf{3}$ was formed in the presence of oxygen: Borsarelii, C. D.; Mischne, M.; La Venia, 
A.; Vieyra, F. E. M. UVA Self-photosensitized oxygenation of $\beta$ ionone. Photochem. Photobiol. 2007, 83, 1313-1318.

(22) $(Z)$-3a was prepared from $(E)-3 \mathbf{a}$ using the following procedure: Singh, K.; Staig, S. J.; Weaver, J. D. Facile synthesis of Z-alkenes via uphill catalysis. J. Am. Chem. Soc. 2014, 136, 5275-5278.

(23) For a similar example, see: Molloy, J. J; Schafer, M.; Weinhold, M.; Morack, T.; Daniliuc, C. G.; Gilmour, R. Boron-Enabled geometric isomerization of alkenes via selective energy-transfer catalysis. Science 2020, 369, 320-306.

(24) Antarafacial [1,5]-hydride shifts are favored in rare cases: Kiefer, E. F.; Tanna, C. H. Alternative electrocyclic pathways. Photolysis and termolysis of dimethylallene dimers. J. Am. Chem. Soc. 1969, 91, 4478-4480.

(25) Littke, A. F.; Fu, G. C. A versatile catalyst for Heck reactions of aryl chlorides and aryl bromides under mild conditions. J. Am. Chem. Soc. 2001, 123, 6989-7000.

(26) We engineered an inexpensive FEP tubing spindle for the purpose of scale up. See the Supporting Information (SI) for details.

(27) The relative stereochemistry of $\mathbf{5 j}$ was confirmed via single crystal X-ray diffraction of a $\mathrm{C} 1$ alcohol derivative. See the Supporting Information (SI) for details.

(28) (1) Liu, Y.-L.; Lindert, S.; Zhu, W.; Wang, K.; McCammon, J. A.; Oldfield, E. Taxodione and Arenarone Inhibit Farnesyl Diphosphate Synthase by Binding to the Isopentenyl Diphosphate Site. Proc. Natl, Acad. Sci., USA 2014, 111, E2530-E2539; (b) Han, S.; Li, X.; Xia, Y.; Yu, Z.; Cha, N.; Malal, S. R.; Han, X.; Oldfield, E.; Zhang, Y. Farnesyl pyrophosphate synthase as a target for drug development: Discovery of natural-product-derived inhibitors and their activity in pancreatic cancer cells. J. Med. Chem. 2019, 62, 10867-10896.

(29) (a) Mori, K.; Matsui, M. Diterpenoid total synthesis XIII: Taxodione, a quinone methide tumor inhibitor. Tetrahedron 1970, 26, 3467-3473; (b) Matsumoto, T.; Ohsuga, Y.; Harada, S.; Fukui, K. Synthesis of taxodione, royleanone, cryptojaponol, and methyl 11hydroxy-12-methoxy-7-oxoabieta-8,11)13-trien-18-oate. Bull. Chem. Soc. Jpn. 1977, 50, 266-272; (c) Matsumoto, T.; Usui, S.; Morimoto, T. A convenient synthesis of $( \pm)$-taxodione, $( \pm)$-ferruginol, and $( \pm)$ sugiol. Bull. Soc. Chem. Jpn. 1977, 50, 1575-1579; (d) Snitman, D. L.; Himmelsbach, R. J.; Haltwanger, R. C.; Watt, D. S. A synthesis of $( \pm)$-cryptojaponol and $( \pm)$-taxodione. Tetrahedron Lett. 1979, 20, 2477-2480; (e) Stevens, R. V.; Bisacchi, G. S. Benzocyclobutenones as synthons for the synthesis of C-11 oxygenated diterpenoids. Application to the total synthesis of $( \pm)$-taxodione. J. Org. Chem. 1982, 47, 2396-2399; (f) Johnson, W. S.; Shenvi, A. B.; Boots, S. G. An approach to taxodione involving biomimetic polyene cyclization. Tetrahedron 1982, 38, 1379-1404; (g) Poierier, D.; Jean, M.; Burnell, R. H. Alternate syntheses of taxodione. Synth. Commun. 1983, 13, 201205; (h) Burnell, R. H.; Jean, M.; Poirier, D. Synthesis of taxodione. Can. J. Org. Chem. 1987, 65, 775-781; (i) Haslinger, E.; Michl, G. Synthesis of $(+)$-taxodione from (-)-abietic acid. Tetrahedron Lett. 1988, 29, 5751-5754; (j) Engler, T. A.; Smapath, U.; Naganathan, S.; Velde, D. V.; Takusagawa, F. A new general synthetic approach to diterpenes: Application to the synthesis of $( \pm)$-taxodione and $( \pm)$ royleanone. J. Org. Chem. 1989, 54, 5712-5727.

(30) This total synthesis begins from commercial geranyl chloride and 2-isopropylveratrole: Harring, S. R.; Livinghouse, T. A concise biomimetic total synthesis of $( \pm)$-taxodione via a $\mathrm{BF}_{3} \bullet \mathrm{MeNO}_{2}$ promoted cationic cascade annulation. J. Chem. Soc., Chem. Commun. 1992, 502-503.

(31) Simmons, E. M.; Sarpong, R. Structure, Biosynthetic Relationships and Chemical Synthesis of the Icetexane Diterpenoids. Nat. Prod. Rep. 2009, 26, 1195-1217.

(32) Thommen, C.; Neuburger, M.; Gademann, K. Collective Syntheses of Icetexane Natural Products Based on Biogenic Hypothesis. Chem. Eur. J. 2017, 23, 120-127.

(33) (a) Simmons, E. M.; Sarpong, R. Ga(III)-Catalyzed Cycloisomerization Strategy for the Synthesis of Icetexane Diterpenoids: Total Synthesis of ( \pm )-Salviasperanol. Org. Lett. 2006, 8, 2883-2886; (b) Majetich, G.; Zou, G.; Grove, J. Total Synthesis of ( \pm )Salviasperanol. Org. Lett. 2008, 10, 85-87.
(34) Yao, M.-L.; Pippin, A. B.; Kabalka, G. W. Deoxygenation of benzylic alcohols using chloroboranes. Tetrahedron Lett. 2010, 51, 853-855.

(35) Dueterm R. K.; Pounds, S. Ring-opening reactions of electrophilic cyclopropanes. J. Org. Chem. 1982, 47, 3174-3177.

(36) The unpurified mixture of deprotected $\mathbf{1 3}$ was concentrated onto $\mathrm{SiO}_{2}$, dissolved in $\mathrm{CHCl}_{3}$, and stirred for $1 \mathrm{~h}$ under a balloon of $\mathrm{O}_{2}$. Filtration of the resulting slurry afforded $\mathbf{8}$ in $>95 \%$ purity.

(37) In principal, $\mathbf{A}$ could arise from the initial Lewis adduct of $\mathbf{1 1}$ via consecutive $[1,2]$ alkyl shifts or a net $[1,3]$ alkyl shift involving a nonclassical carbocation: Hong, Y. J.; Giner, J.-L.; Tantillo, D. J. Bicyclobutonium ions in biosynthesis - Interconversion of cyclopropylcontaining sterols from orchids. J. Am. Chem. Soc. 2015, 137, 20852088.

(38) Cossy, J.; Furet, N. Photochemical ring opening of cyclopropyl ketones induced by electron transfer. Tetrahedron Lett. 1993, 34, $8107-8110$.

(39) For an overview of the cyclocitrinols, see: Wu, J.; Liu, J.; Fan, J.H.; Xie, Z.-D.; Qin, H.; Li, C.-C. Evolution of routes for asymmetric total synthesis of cyclocitrinol enabled by type II [5+2] cycloaddition. Chin. J. Chem. 2021, 39, 1247-1254. 